\title{
The LBTI Fizeau imager - II. Sensitivity of the PSF and the MTF to adaptive optics errors and to piston errors
}

\author{
F. Patru, ${ }^{1,2 \star}$ S. Esposito, ${ }^{2}$ A. Puglisi, ${ }^{2}$ A. Riccardi, ${ }^{2}$ E. Pinna,${ }^{2}$ C. Arcidiacono, ${ }^{3}$ \\ J. Antichi, ${ }^{4,2}$ B. Mennesson, ${ }^{5}$ D. Defrère, ${ }^{6,7}$ P. M. Hinz ${ }^{7}$ and J. M. Hill ${ }^{7}$ \\ ${ }^{1}$ PSL Research University, CNRS, Sorbonne Universités, UPMC Univ. Paris 06, Univ. Paris Diderot, Sorbonne Paris Cité, Observatoire de Paris, LESIA, 5 \\ place Jules Janssen, F-92195 Meudon cedex, France \\ ${ }^{2}$ INAF Osservatorio Astrofisico di Arcetri, 5 Largo Enrico Fermi, I-50125 Firenze, Italy \\ ${ }^{3}$ INAF Osservatorio Astronomico di Bologna, 1 Via Ranzani, I-40127 Bologna, Italy \\ ${ }^{4}$ Airbus Defense and Space GmbH, Space Systems, Robert Koch Str. D-82024 Taufkirchen, Munich, Germany \\ ${ }^{5}$ Jet Propulsion Laboratory, 4800 Oak Grove Drive, CA 91109, Pasadena, United States \\ ${ }^{6}$ Space Sciences, Technologies and Astrophysics Research Institute, University of Liége, 7 place du 20-Aožt, B-4000 Liége, Belgium \\ ${ }^{7}$ Steward Observatory, University of Arizona, 933 N. Cherry Avenue, 85721, Tucson, United States
}

Accepted 2017 August 3. Received 2017 July 26; in original form 2017 April 5

\begin{abstract}
We show numerical simulations with monochromatic light in the visible for the LBTI Fizeau imager, including opto-dynamical aberrations due here to adaptive optics (AO) errors and to differential piston fluctuations, while other errors have been neglected. The achievable Strehl by the LBTI using two AO is close to the Strehl provided by a single standalone AO system, as long as other differential wavefront errors are mitigated. The LBTI Fizeau imager is primarily limited by the $\mathrm{AO}$ performance and by the differential piston/tip-tilt errors. Snapshots retain high-angular resolution and high-contrast imaging information by freezing the fringes against piston errors. Several merit functions have been critically evaluated in order to characterize point spread functions and the modulation transfer functions for highcontrast imaging applications. The LBTI Fizeau mode can provide an image quality suitable for standard science cases (i.e. a Strehl above 70 percent) by performing both at a time: an $\mathrm{AO}$ correction better than $\approx \lambda / 18 \mathrm{RMS}$ for both short and long exposures, and a piston correction better than $\approx \lambda / 8$ RMS for long exposures or simply below the coherence length for short exposures. Such results, which can be applied to any observing wavelength, suggest that $\mathrm{AO}$ and piston control at the LBTI would already improve the contrast at near- and mid-infrared wavelengths. Therefore, the LBTI Fizeau imager can be used for high-contrast imaging, providing a high-Strehl regime (by both AO systems), a cophasing mode (by a fringe tracker) and a burst mode (by a fast camera) to record fringed speckles in short exposures.
\end{abstract}

Key words: instrumentation: adaptive optics - instrumentation: interferometers - methods: numerical - techniques: high angular resolution - techniques: interferometric.

\section{INTRODUCTION}

The Large Binocular Telescope Interferometer (LBTI) (Angel \& Woolf 1997; Angel et al. 1998; Herbst 2003; Hill et al. 2014; Hinz et al. 2014; Hinz et al. 2016) is able to provide fringes since 2010, complemented with adaptive optics (AO)-correction since 2012 (Hinz et al. 2012; Leisenring et al. 2012) and with piston compensation since 2013 (Defrère et al. 2014). We have shown by numerical

^E-mail: fabienpatru@gmail.com simulations that the LBTI Fizeau imager (Patru et al. 2017) can significantly improve the raw contrast by means of two first light adaptive optics (FLAO) systems (Esposito et al. 2010). Compared to a single standalone 8-m LBT aperture, the 23-m LBTI Fizeau imager can provide a gain in sensitivity (by a factor of 4 ), a gain in angular resolution (by a factor of 3 ) and, as well, a gain in raw contrast (by a factor of 2 to 1000 varying over the AO-corrected field of view). The contrast gain averaged across the AO-corrected field of view is improved by a factor of 2 in contrast in long exposures and by a factor of 10 in contrast in short exposures (i.e. in exposures respectively longer or shorter than the coherence time). 
In other words, using the FLAO systems at high-Strehl regime on to the LBTI instead of a single LBT improves at a minimum twice the contrast at comparable inner working angles.

We have performed here numerical simulations for the LBTI Fizeau imager including opto-dynamical aberrations, due here to $\mathrm{AO}$ errors and to differential piston fluctuations. For each frame, two independent FLAO phase screens - reconstructed from FLAO on-sky data - are introduced in the complex pupil function with a random piston reproducing realistic injection of the two separated beams into the beam combiner. Given an incoming wavefront with aberrations, we deduce the imaging properties by computing the point spread function (PSF) and the modulation transfer function (MTF) for short or long exposures. Those simulations have been described more in details in previous papers (Patru et al. 2016a,b).

In this paper, we focus on the main aberrations affecting the performance of the LBTI (Section 2). We consider separately the residual wavefront errors of the FLAO systems and the differential piston between the two telescopes (Section 3), which are supposed to be uncorrelated here. We study the main properties of the PSF and the MTF of the LBTI (Section 3.1), by assuming either increasing AO RMS errors and no residual piston (Section 3.2) or increasing piston RMS errors and perfect AO correction (Section 3.3). We critically evaluate various merit functions used to characterize the PSFs and the MTFs, in order to understand which ones are most useful to describe the high-contrast imaging performance of a given aberrated wavefront (Section 4). Finally, we discuss on the requirements and on the performance of the LBTI equipped with both FLAO systems, as well as with piston/tip-tilt correction systems (Section 5).

\section{MAIN SOURCES OF ABERRATIONS}

According to the LBT/LINC-NIRVANA error budget (Herbst et al. 2005), a 1-2.5 micron interferometric imaging instrument operating in Fizeau beam combination mode (Herbst et al. 2004), the primary contributors reducing the on-axis performance are imperfect AO correction (across each sub-aperture) and uncorrected atmospheric piston (between both sub-apertures). The beauty of the LBTI Fizeau mode is that in principle high-contrast imaging is also achievable off-axis, only under the condition that field effects are under control (e.g. differential plate-scale, geometric distortion, differential isoplanatic angles, etc.). We neglect here such wide-field $\mathrm{AO}$ errors and we consider the imaging capabilities across the inner part of the AO-corrected field of view (typically a field of view of $32 \lambda / 23 \mathrm{~m} \approx 200$ mas at $750 \mathrm{~nm}$ ).

We concentrate our study on to opto-dynamical aberrations, due here to AO errors and to differential piston fluctuations, while neglecting any other instrumental effects (e.g. vibrations, misalignments, non-common path aberrations, surface quality of the optical components, etc.). We assume favourable conditions of observations with a good seeing and negligible atmospheric effects between the two 8-m telescopes. We consider that the LBTI facility is equipped with efficient FLAO systems at high Strehl and a fast piston/tiptilt mirror. We have performed monochromatic calculations in the visible $(\lambda=750 \mathrm{~nm})$, but it can be applied to any observing wavelength, since performances are shown in term of root mean square (RMS) wavefront error in wavelength unit. The effects of the spectral bandwidth is beyond the scope of this study. Despite of all those assumptions, the results presented here are relevant for a high-Strehl AO regime in good atmospheric conditions providing narrow-band filters.

\subsection{AO errors from each sub-aperture}

Poor atmospheric conditions and faint guide stars will seriously mitigate the AO performance. The AO correction is optimum for the low-order aberrations, decreases monotonically up to the AO cut-off frequency and is null for high-order aberrations beyond the cutoff. Besides, the main errors contributions of a single AO system are due to the low-order aberrations. The individual tip-tilt is, by far, more significant than the contribution of higher order aberrations: focus, astigmatism, etc. (Noll 1976).

Consequently, differential tip-tilt jitters between the two subapertures are unseen by both $\mathrm{AO}$ due to the independency of the two AO loop control systems. Moreover, the Fizeau mode is more sensitive to differential tip-tilt than to individual tip-tilt. An individual tip-tilt is produced by a $8-\mathrm{m}$ turbulent cell which is not perfectly compensated by a single AO system. A differential tip-tilt is generated over a worse $23-\mathrm{m}$ turbulent cell corresponding to the longest baseline, requiring an additional sensing and correction system of the differential tip-tilt. Therefore, the LBTI Fizeau imager is primarily limited by the AO performance and, as well, by the differential tip-tilt between the two sub-apertures.

\subsection{Piston errors between the two sub-apertures}

The performance of the LBTI Fizeau imager is also affected by the differential piston fluctuations. Time-varying piston can arise from numerous effects (Bailey et al. 2014; Defrère et al. 2014). The atmospheric effect is, by far, one of the main contribution to piston errors. In particular, a tip-tilt across large turbulent cells of $23-\mathrm{m}$ is translated into a piston between the two LBTI sub-apertures. The AO systems provide flat wavefronts but cannot control the relative time of arrival of the wavefronts at the two telescopes, so that a fringe tracking system is required. Therefore, the performance of the LBTI Fizeau imager is also affected by the piston errors generated by the atmosphere which are unseen by both AO systems and which may not be well compensated by fringe tracking.

\subsection{Piston and tip-tilt errors due to vibrations}

Vibrations of a single LBT telescope occur in a frequency range from 0 to $50 \mathrm{~Hz}$ and an amplitude range from 10 to 50 mas RMS. In particular, the mirror M2 - mounted high above the primary mirror tends to vibrate strongly, leading to low-order aberrations of piston and tip-tilt. While tip-tilt can in theory be corrected by a fast AO control loop, the AO is not sensible to piston. Moreover, classical AO systems cannot completely suppress the high-frequency vibrations which would require a very short exposure time of the wavefront sensor, preventing the use of faint natural guide stars.

Different strategies have been proposed in the literature for minimizing the effects of telescope vibrations on to the differential piston and tip-tilt values when observing faint guide stars. Some approaches have been tested at the LBT facility for estimating the fluctuations with accelerometers and for compensating them with a piston/tip-tilt mirror, like the optical vibration measurement system (OVMS; Böhm et al. 2014, 2016; Defrère et al. 2016a) and the disturbance feedforward (DFF) control (Glück, Pott \& Sawodny 2017). The LBT is now equipped with a system able to calculate piston and tip-tilt values for all its main mirrors in real time. The differential piston induced by telescope vibrations can be reduced by a factor of about 5 below $200 \mathrm{~nm}$ RMS. The AO Strehl can be increased by a factor of more than 2 . 
Actually, what currently limits the LBTI performance are the vibrations. We believe that such accelerometer-based techniques will enable to improve the $\mathrm{AO}$ performance and the fringe tracking efficiency against mirror vibrations on both telescopes. The vibration's effects have been neglected in this paper but will be accounted in forthcoming studies.

\section{LBTI RECOMBINATION OF TWO ON-SKY FLAO WAVEFRONTS}

\subsection{PSF and MTF of the LBTI}

The PSF equals to squared modulus of the Fourier transform of the complex pupil function. The LBTI provides an in-between PSF, combining both diffractive and interferometric properties. The fringes appear within an Airy envelope, due to the double-aperture nature of the LBTI. The theoretical PSF can be written as the product of a diffraction pattern and an interference pattern. The diffraction pattern is the sum of the two Airy functions delivered by each sub-aperture. The interference pattern is a fringe cosine function obtained by combining both sub-apertures. The diffraction and interference patterns produce respectively rings and fringes which are alternatively bright and dark. The bright rings and fringes are features located on to a side-lobe in the PSF profile, where the high irradiance mitigates the achievable contrast. The dark rings and fringes are located into a valley (between two side-lobes), where the low irradiance enables locally a high-contrast gain. A huge contrast gain in narrow zones can be achieved when both a dark fringe and a dark ring overlap.

The shape and size of the PSF structure are directly related the shape and size of the LBTI aperture. The entrance pupil of the LBTI is made of two primary mirrors of diameter $D=8.4 \mathrm{~m}$ having a central obscuration of diameter $0.9 \mathrm{~m}$, a centre-to-centre separation of $B=14.4 \mathrm{~m}$ and a maximum baseline equal to $B_{\max }=B+D=22.8 \mathrm{~m}$. The maximum baseline $B_{\max }=22.8 \mathrm{~m}$ imposes the angular resolution in the PSF, i.e. the width of the central lobe $\lambda / B_{\max }\left(\lambda / B_{\max }=6.8\right.$ mas for $\left.\lambda=750 \mathrm{~nm}\right)$. The LBTI mounting baseline $B=14.4 \mathrm{~m}$ fixes the fringe period $\lambda / B$ in the interferometric PSF pattern. The sub-aperture diameter $D=8.4 \mathrm{~m}$ determines the Airy envelope extent $2.44 \lambda / D$ in the diffractive PSF pattern.

The MTF is the modulus of the Fourier transform of the PSF yielding to a central peak surrounded by two identical and weaker peaks. The peak widths equal to $2 D / \lambda$ and the peak centres are separated by a distance $B / \lambda$. The central peak contains the lowangular resolution information, i.e. the angular frequencies seen by the short baselines spanning two points within each 8.4-m subaperture. The lateral peaks contain the high-angular resolution information, i.e. the angular frequencies sampled by the long baselines spanning both sub-apertures (Herbst et al. 2008). The (u,v) plane coverage is complete along the baseline axis thanks to the small overlap of the high- and low-angular frequency peaks. The maximum baseline $B_{\max }=22.8 \mathrm{~m}$ imposes the MTF extent covering the $(\mathrm{u}, \mathrm{v})$ plane, i.e. the highest angular frequency sampled in the image plane and equal to $B_{\max } / \lambda$.

\subsection{Sensitivity to adaptive optics errors RMS}

The aberrations of the AO wavefronts disturb both the PSF and the MTF of the LBTI. The achievable Strehl by the LBTI using two AO is close to the Strehl provided by a single standalone AO system, as long as other differential wavefront errors are mitigated. The
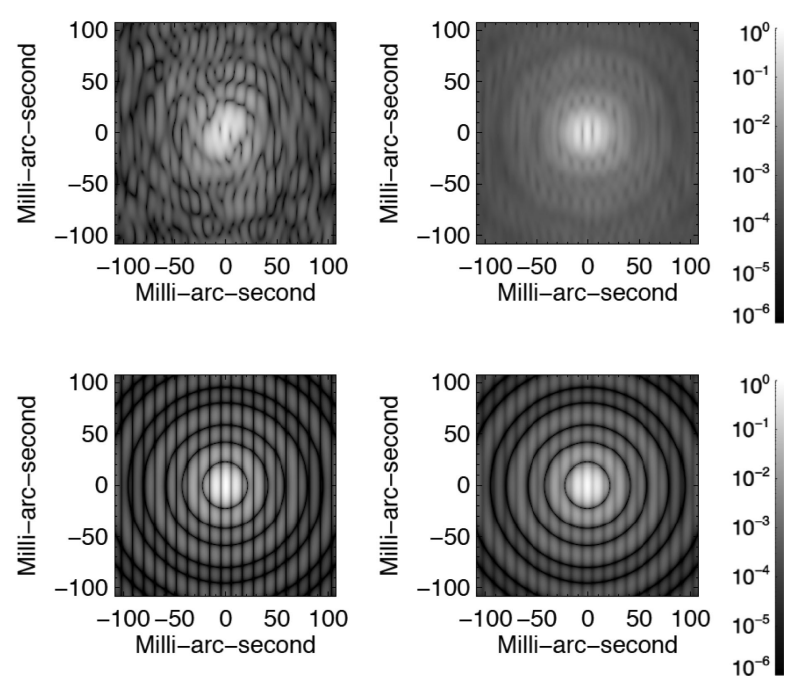

Figure 1. PSF distributions with only AO errors (top) or with only piston errors (bottom), given a residual RMS of $\lambda / 8$. Short exposure (left) and long exposure (right). Monochromatic light (at $\lambda=750 \mathrm{~nm}$ ). Other figures with various RMS are shown in (Patru et al. 2016b).

contrast in the PSF is primarily limited by the AO performance, whatever the exposure time (Fig. 1 and Fig. 3). In the presence of residual AO aberrations, the diffraction-limited PSF, made of a fringed Airy pattern, reduces to an AO-corrected PSF, made of a central core surrounded by a speckle halo. Regarding the fringe pattern against $\mathrm{AO}$ errors, good AO compensation is required in long exposures, but not in short ones. For poor $\mathrm{AO}$ correction, the fringes and the Airy rings are blurred in long exposures, yielding a diffuse speckle halo. On the opposite, snapshots produce a fringed speckle halo, where fringes are still visible across the AO-corrected field of view. Even if the diffractive rings are destroyed by AO errors, snapshots enable to freeze the fringed speckle halo, as stated by speckle imaging and speckle interferometry (Labeyrie 1970; Patru et al. 2017).

The contrast in the PSF is also affected by the differential errors between the two sub-apertures, mainly the differential tip-tilt. The individual tip-tilts of each AO system induces differential tip-tilts of the sub-beams and differential shifts of the sub-images, so that the diffraction envelopes no longer overlap properly, limiting the extent of the coherent area containing the fringes. The differential tip-tilt can be neglected if the separation between the envelopes centres do not exceed a small fraction of the envelope width $\lambda / D$. The coherent area where fringes occur gets an ovoidal shape orthogonal to the tip-tilt direction for short exposures, while its circular shape is recovered by average for long exposures. For large differential tip-tilt, when the shifts of the envelopes become larger than their widths (i.e. RMS $>\lambda$ ), the decorrelation into two sub-PSFs prevents fringe occurrence and reduces the maximum of the LBTI PSF by a factor of 4 compared to the theoretical one.

Short and long exposures of the MTFs behave similarly (Fig. 2 and Fig. 4). Both the central and lateral peaks are disturbed by the residual aberrations of the AO-corrected wavefront (mainly the differential tip-tilt).

\subsection{Sensitivity to differential piston errors RMS}

The contrast in the PSF is disturbed in a different manner by piston fluctuations, assuming here no AO residual errors (Fig. 1 and Fig. 3). 

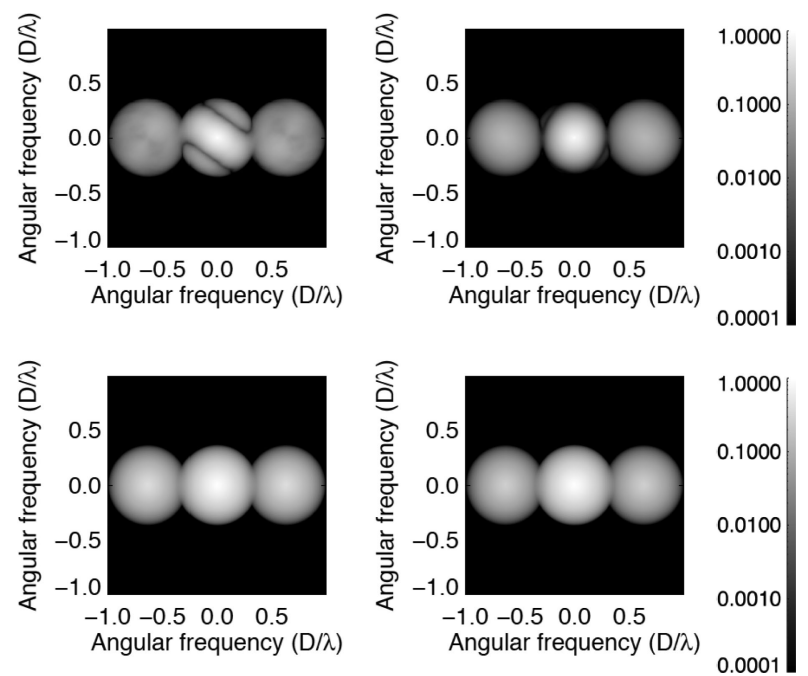

Figure 2. MTF distributions with only AO errors (top) or with only piston errors (bottom), given a residual RMS of $\lambda / 8$. Short exposure (left) and long exposure (right). Monochromatic light (at $\lambda=750 \mathrm{~nm}$ ). Other figures with various RMS are shown in (Patru et al. 2016b).

The fringe motion decreases the fringe contrast for long exposures, but not for short ones.

During snapshots (given an exposure time significantly shorter than the coherence time of the turbulence), the piston varies over a much larger time-scale, so that the fringe motion is frozen. Thus, a snapshot preserves the fringe contrast against piston errors, assuming a piston RMS lower than the coherence length. The short exposure PSF is still a fringed Airy pattern, where the rings are unaffected and the fringes are shifted and well-contrasted across the whole field of view.

During long exposures (significantly longer than the coherence time), the piston varies over a much shorter time-scale, blurring the fringes and attenuating their contrast. The fringe contrast can be maintained only if the piston RMS remains lower than a fraction of the wavelength. For piston RMS larger than the wavelength during long exposures (and, as well, for piston RMS larger than the coherence length during short exposures), the fringes in the Airy pattern are completely blurred while rings are not disturbed. The PSF turns into the superposition of the two Airy patterns, without any more fringes, reducing the maximum of the PSF by a factor of 2 compared to the theoretical one.

Short exposure MTFs are not affected by piston fluctuations (Fig. 2 and Fig. 4). For long exposure MTFs, the central peak is preserved, whereas the amplitude of the lateral peaks decreases as the piston errors increases.

It is worth noting that random pistons are generated in the sequence of frames by using a Gaussian distribution of zero mean with a given RMS expressed as a fraction of the wavelength. This approach is enough when considering many exposures that are sufficiently shorter or longer than the coherence time. To go further, our main result can be used to define the right exposure times (below the coherence time) for defining a burst mode when aiming for high contrast. For that purpose, instead of using random pistons, one needs initially to derive the atmospheric differential piston from the power spectrum of an interferometer (Buscher et al. 1995), but it is beyond the scope of this study.

\section{MERIT FUNCTIONS}

In this section, we evaluate several merit functions (Fig. 5) in order to characterize the PSFs and the MTFs structures changing as a function of the wavefront aberrations (Fig. 6 and Fig. 7).

\subsection{Interferometric Strehl of the PSF}

The interferometric Strehl (Herbst et al. 2005) - or peak Strehl (McCarthy et al. 2000) - ratio equals to the maximum of the aberrated PSF divided by the maximum of the unaberrated PSF, i.e. the ratio of the maximum irradiance of the central lobe over its theoretical value:

$S_{r}=\left(I_{\max }\right) /\left(I_{\max }^{T h}\right)$

Contrary to the traditional Strehl ratio which is only suitable onaxis, the interferometric Strehl remains valid out-of-axis since it does not depend on the shift of the fringes produced by a piston during snapshots. Thus, the interferometric Strehl accounts for the fact that the high-angular resolution information delivered by the interferometer is preserved in snapshots despite of fringe displacements (Fig. 6). A reduction of 15 percent of the interferometric Strehl is due to a residual $\mathrm{AO}$ wavefront $\mathrm{RMS} \approx \lambda / 18 \approx 40 \mathrm{~nm}$ for both short and long exposures or to a residual piston $\mathrm{RMS} \approx \lambda / 8 \approx$ $100 \mathrm{~nm}$ for long exposures. A reduction of 50 per cent occurs for $\mathrm{AO}$ $\mathrm{RMS}>\lambda / 8 \approx 100 \mathrm{~nm}$ or for piston RMS $>\lambda / 2 \approx 400 \mathrm{~nm}$. Piston errors do not affect the interferometric Strehl during snapshot.

\subsection{Contrast in the PSF}

The PSF of the LBTI is made of rings and fringes alternatively bright or dark (Section 3.1). Contrary to the periodic location of the fringes, the rings are not regularly distributed due to the central obscuration on each LBT aperture. Therefore, fringes and rings features are intricate and overlap at specific radial distances to form the PSF structure. In the X profile parallel to the baseline direction (Fig. 3), we can distinguish amongst the local minima the valleys formed by the zeros in the Airy pattern (dark rings) and the valleys induced between the fringes (dark fringes).

The contrast is defined as the normalized PSF irradiance at a given parallactic angle $\theta$ and a given radial distance $r$ from the PSF centre, i.e. the ratio of the off-axis irradiance at the polar coordinate $(r, \theta)$ over the theoretical maximum irradiance:

$C(r, \theta)=(I(r, \theta)) /\left(I_{\max }^{T h .}\right)$

The contrast behaves differently depending on the exposure time, on the location in the field of view and on the sources of aberrations. Despite of various behaviours rapidly changing from point to point across the field, some singular points or regions might be considered to illustrate the $\mathrm{AO}$ and piston effects on to the fringes and rings structures. We show (Fig. 6) the contrast circularly averaged at 59 mas in all the directions which is located along a dark ring in the $360^{\circ}$ profiles. We consider also the local contrast at 42, 48, 59 and 87 mas in the baseline direction. The contrast at 48 and 59 mas in the $\mathrm{X}$ profile corresponds to a dark fringe, whereas the contrast at 87 and 42 mas corresponds to a bright fringe, overlapping either a bright ring or a dark ring, respectively. Snapshots cannot only mitigate the piston errors but also the AO contribution by recovering some dark features (e.g. at 42 and 48 mas on the $\mathrm{X}$ axis). A huge contrast where dark fringes and rings overlap is only achievable in a highStrehl AO regime. A contrast of $10^{-4}$ at 59 mas on the $X$ axis can be directly attained providing AO RMS below $\lambda / 32 \approx 20 \mathrm{~nm}$ (Fig. 6). 

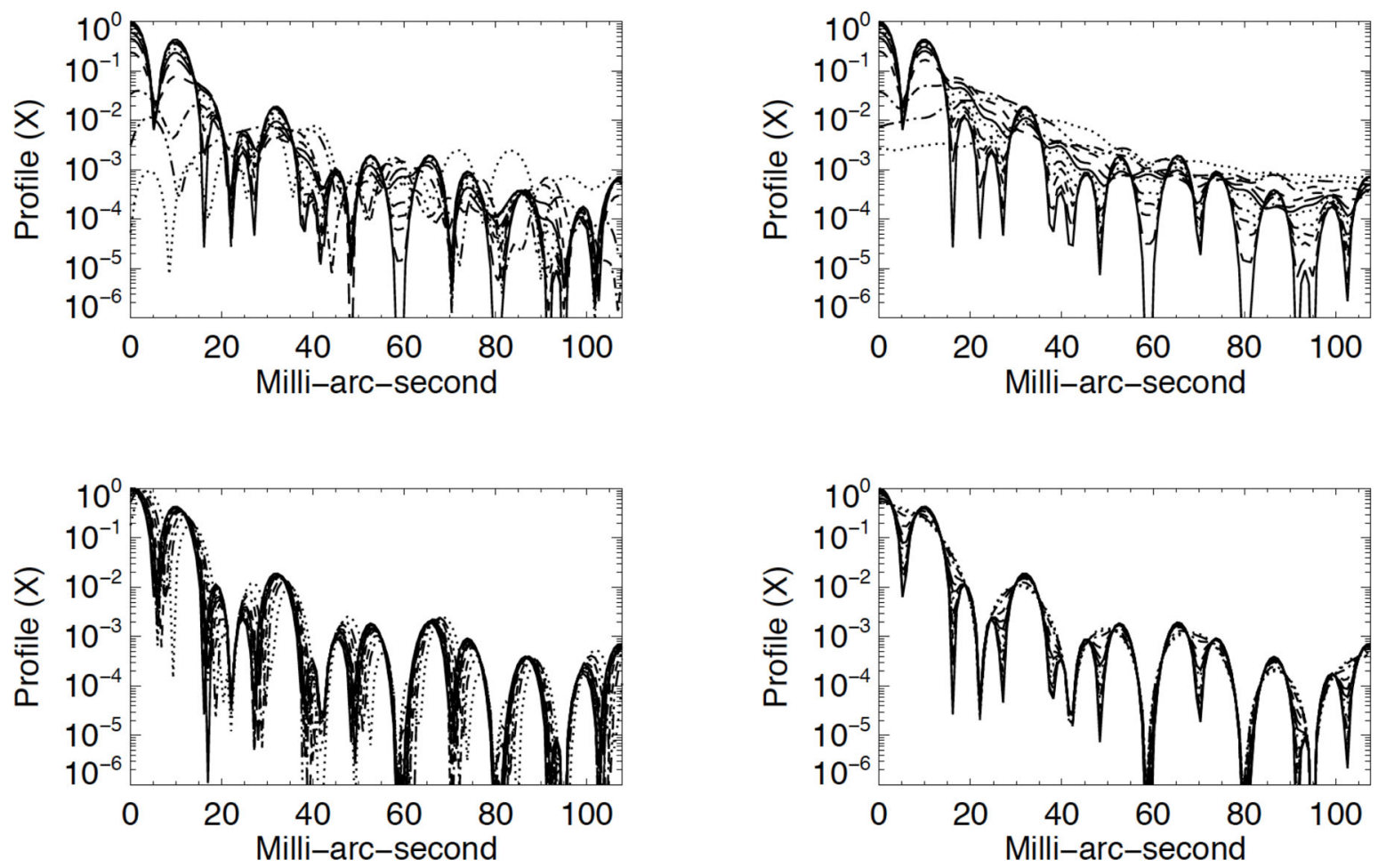

Figure 3. PSF profiles with only AO errors (top) or with only piston errors (bottom), given an RMS equal to 0 (solid line), $\lambda / 64$ (long dash), $\lambda / 32$ (dash), $\lambda / 24$ (dash dot), $\lambda / 18$ (dash dot dot), $\lambda / 12$ (dot), $\lambda / 10$ (solid line), $\lambda / 8$ (long dash), $\lambda / 6$ (dash), $\lambda / 4$ (dash dot), $\lambda / 3$ (dash dot dot), $\lambda / 2$ (dot). Short exposure (left) and long exposure (right).
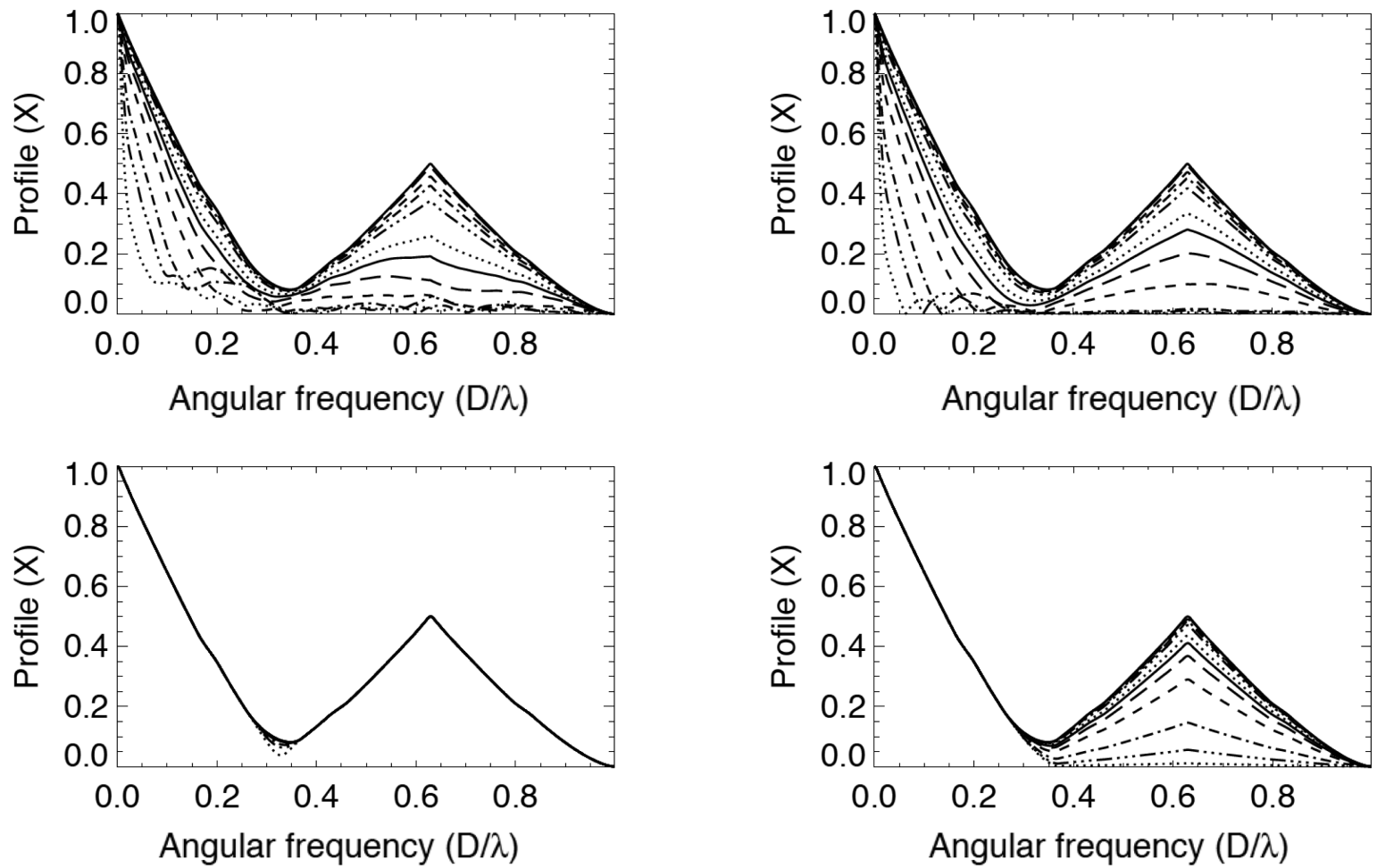

Figure 4. MTF profiles with only AO errors (top) or with only piston errors (bottom). Same legend as in Fig. 3.

Furthermore, the dark features in the PSF are more sensitive than the bright features to aberrations. It is due to the peaked shape of a valley as opposed to the smoothed shape at the top of a side-lobe (cross-section of a ring or a fringe). The speckle halo first fills the valleys before altering the side-lobes in the PSF. The parasitic light is mostly redistributed from the bright rings to the dark rings by $\mathrm{AO}$ errors and from the bright fringes to the dark fringes by piston errors (Fig. 6). 

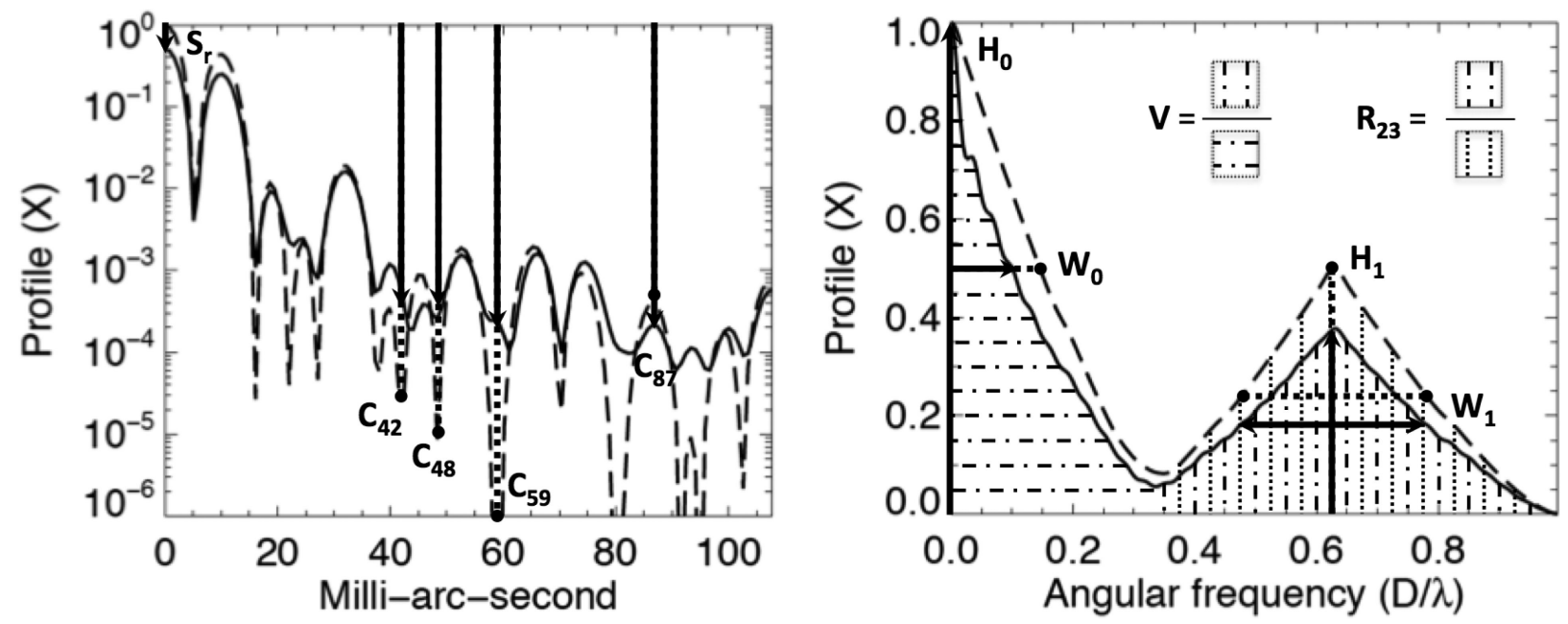

Figure 5. Merit functions definition related to the structures of the theoretical (long-dashed line) and aberrated (solid line) PSF (left) and MTF (right). For the PSF: the interferometric Strehl $S_{r}$, the contrast at a given radial distance $C(r)$. For the MTF: the visibility $V$, the $R_{23}$ quantity, the height $H_{0} \& H_{1}$ and width $W_{0} \& W_{1}$ of the central and lateral frequency peaks. The bold arrows indicate the theoretical (dot line) and aberrated (solid line) criteria defining $S_{r}, C(r), H_{0}$, $H_{1}, W_{0}$ and $W_{1}$. The areas containing dotted-lines and dashed-dotted-lines denote $V$ and $R_{23}$.

\subsection{Interferometric visibility of the MTF}

The visibility $V$ equals to the ratio of the integral in the two MTF lateral peaks over the integral in the MTF central peak:

$V=2 \frac{\int_{0}^{D / 2} \operatorname{MTF}_{[B, 0]}(\rho) \mathrm{d} \rho}{\int_{0}^{D / 2} \operatorname{MTF}_{[0,0]}(\rho) \mathrm{d} \rho}$

where $\rho$ is the radial coordinate in the MTF. A reduction of 15 per cent of the visibility is due to a residual $\mathrm{AO}$ wavefront $\mathrm{RMS} \approx$ $\lambda / 8 \approx 100 \mathrm{~nm}$ and $\approx \lambda / 6 \approx 130 \mathrm{~nm}$ for short and long exposures, respectively, or to a residual piston $\mathrm{RMS} \approx \lambda / 12 \approx 70 \mathrm{~nm}$ for long exposures. A reduction of 50 per cent occurs for AO RMS $>\lambda / 4 \approx$ $200 \mathrm{~nm}$ or for piston RMS $>\lambda / 6 \approx 130 \mathrm{~nm}$. Piston errors do not affect the visibility during snapshot.

The visibility mainly accounts for the bright fringe contrast within the central Airy disc, whereas it is almost insensitive to the faint fringed speckles in the AO-corrected field of view. Therefore, the visibility should not be used as a merit function for highcontrast imaging applications. However, maximizing the visibility is equivalent to maximizing the interferometric Strehl (McCarthy et al. 2000).

\section{4 $R_{23}$ quantity of the MTF}

The $R_{23}$ quantity equals to the ratio of the integral in the lateral MTF peaks over its theoretical value (Egner, Herbst \& Arcidiacono 2008):

$R_{23}=\frac{\int_{0}^{D / 2} \operatorname{MTF}_{[B, 0]}(\rho) \mathrm{d} \rho}{\int_{0}^{D / 2} \operatorname{MTF}_{[B, 0]}^{T h .}(\rho) \mathrm{d} \rho}$

$R_{23}$ quantifies the amount of high-angular resolution information that is retained in the aberrated image. A reduction of 15 percent of the $R_{23}$ quantity is due to a residual AO wavefront $\mathrm{RMS} \approx \lambda / 18$ $\approx 40 \mathrm{~nm}$ for both short and long exposures or to a residual piston $\mathrm{RMS} \approx \lambda / 12 \approx 70 \mathrm{~nm}$ for long exposures (Fig. 7). A reduction of 50 per cent occurs for AO RMS $>\lambda / 8 \approx 100 \mathrm{~nm}$ or for piston RMS $>\lambda / 6 \approx 130 \mathrm{~nm}$. Piston errors do not affect the $R_{23}$ quantity during snapshot. The LBT/LINC-NIRVANA error budget (Herbst et al. 2005) is in agreement with these results.

\subsection{Height and width of the peaks of the MTF}

The heights of the MTF central and lateral peaks - of index 0 and 1 - are defined by their maximum normalized by the maximum of the central MTF peak:

$H_{0}=1$

$H_{1}=\left(\operatorname{MTF}_{[B, 0] \max }\right) /\left(\operatorname{MTF}_{[0,0] \max }^{T h .}\right)$

The widths of the MTF central and lateral peaks - of index 0 and 1 - equal to the full width at half-maximum (FWHM) normalized by the theoretical FWHM:

$$
\begin{aligned}
W_{0} & =\left(\operatorname{FWHM}\left(\operatorname{MTF}_{[0,0]}\right)\right) /\left(\operatorname{FWHM}\left(\operatorname{MTF}_{[0,0]}^{T h .}\right)\right) \\
W_{1} & =\left(\operatorname{FWHM}\left(\operatorname{MTF}_{[B, 0]}\right)\right) /\left(\operatorname{FWHM}\left(\operatorname{MTF}_{[B, 0]}^{T h .}\right)\right)
\end{aligned}
$$

Both piston and $\mathrm{AO}$ errors reduce the lateral peak height in the same manner as the $R_{23}$ quantity (Fig. 7), those criteria characterizing respectively the intensity and the energy of the peaks. Piston errors decrease only the lateral peak height for long exposures, but not for short ones, and have strictly no effect on to the peak widths whatever the exposure time. In opposition, AO errors decrease the central peak width and increase the lateral peak width for both short and long exposures. A reduction of 15 per cent of the FWHM is due to $\mathrm{AO}$ errors of $\mathrm{RMS} \approx \lambda / 18 \approx 40 \mathrm{~nm}$. A reduction of 50 per cent occurs for AO RMS $>\lambda / 8 \approx 100 \mathrm{~nm}$.

Finally, the central peak width and the lateral peak height of the MTF may be used as additional criteria to disentangle the effects due either to piston or to AO aberrations, either in short or in long exposures. The central peak width and the lateral peak height are both reduced by AO errors for both short and long exposures. Only the lateral peak height is disturbed by piston errors for long exposures, but not for short ones. The lateral peak width remains unaffected by piston whatever the exposure time. Therefore, in 

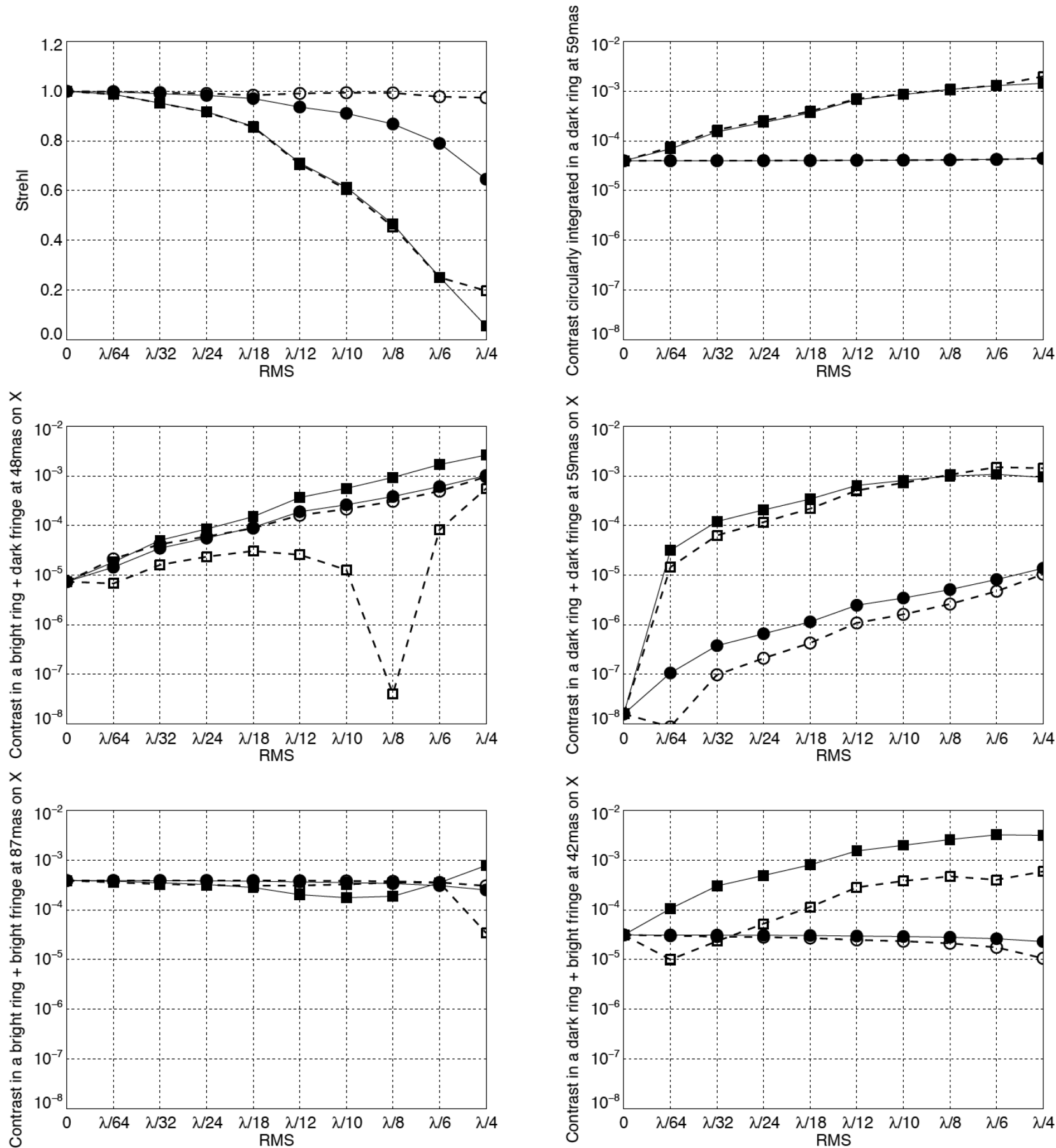

Figure 6. Merit functions for the PSF: interferometric Strehl (top left), contrast circularly integrated in a dark ring at 59 mas (top right), contrast on X in a bright ring and a dark fringe at 48 mas (middle left), contrast on the $\mathrm{X}$ axis in a dark ring and a dark fringe at 59 mas (middle right), contrast on $\mathrm{X}$ in a bright ring and a bright fringe at 87 mas (bottom left), contrast on $\mathrm{X}$ in a dark ring and a bright fringe at 42 mas (bottom right). AO wavefront errors (square symbol) or piston errors (circular symbol) for different RMS values. Short exposure (empty symbol, dashed line) and long exposure (filled symbol, solid line).

short exposures, one can measure either the central peak width or the lateral peak height to estimate the AO aberrations. In long exposures, one can measure the central peak width to estimate the $\mathrm{AO}$ contribution and the lateral peak height to deduce subsequently the piston contribution.

\section{DISCUSSION}

\subsection{Relevance of the merit functions}

Several merit functions have been critically evaluated in order to characterize PSFs and the MTFs for high-contrast imaging 

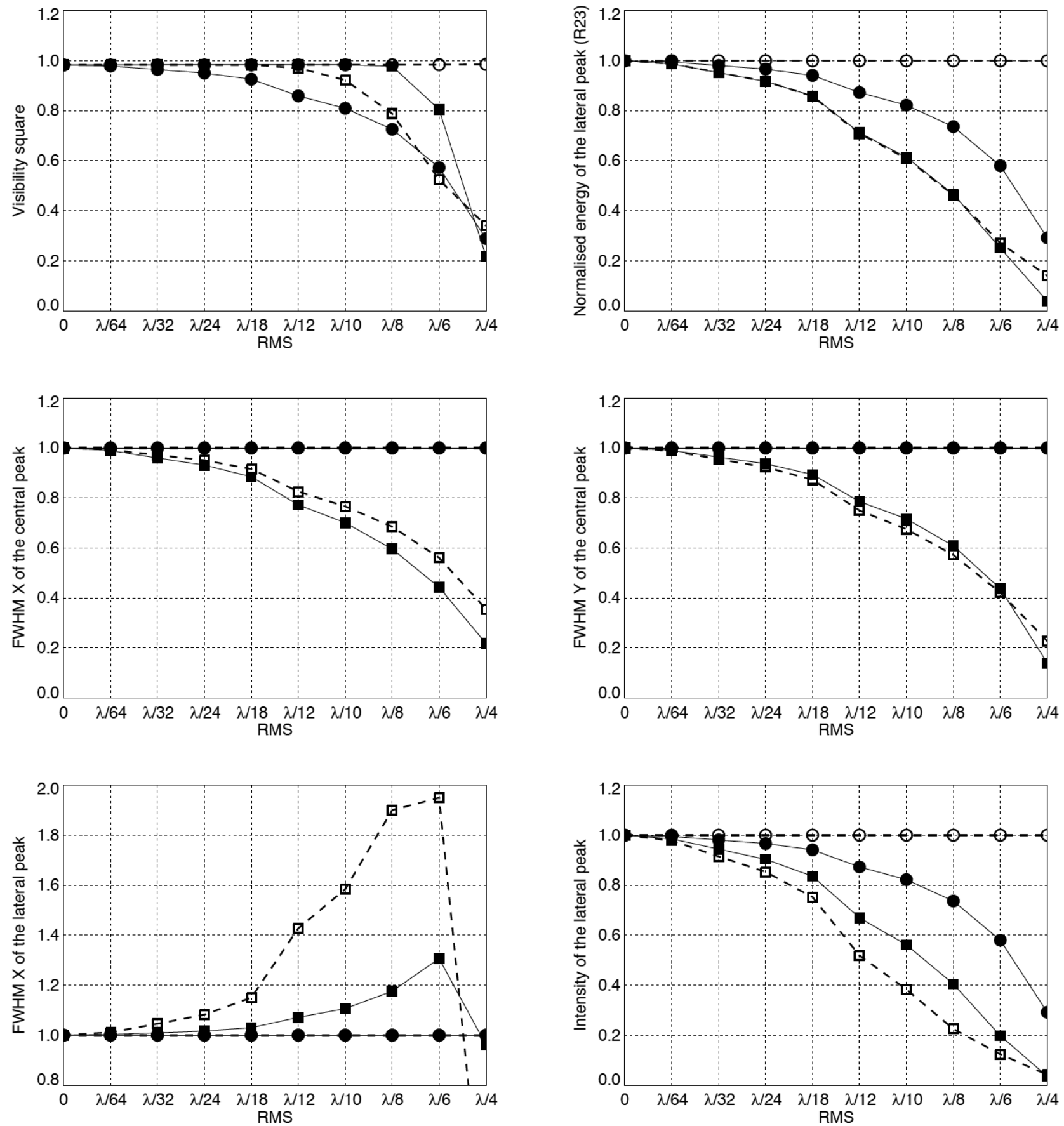

Figure 7. Merit functions for the MTF: interferometric visibility (top left), $R_{23}$ quantity (top right), central peak width in X (middle left), central peak width in $\mathrm{Y}$ (middle right), lateral peak width in X (bottom left), lateral peak height (bottom right). Same legend as in Fig. 6.

applications with the LBTI Fizeau imager (Table 1). The contrast in the PSF behaves differently depending on the exposure time, on the location in the field of view and on the sources of aberrations, so that such criterium may be used with caution. The visibility should not be used as a merit function for high-contrast imaging applications.

The interferometric Strehl and the $R_{23}$ quantity appears as two relevant merit functions which behave similarly, $R_{23}$ being even more sensitive than the Strehl to image blurring during long exposures. The central peak width and the lateral peak height of the MTF may be used as additional criteria to disentangle the effects due to either
AO aberrations or piston errors, in either short or long exposures (Section 4.5). By computing those merit functions for each frame, we can select the ones having a higher quality in order to improve the final signal-to-noise ratio.

In particular, frames with a significant differential tip-tilt between the two beams should be discarded by rejecting the ones having fringes in the central peak of the MTF. Differential tip-tilt aberrations produces fringes in the central MTF peak which are linear in short exposures and circular in long exposures (Fig. 2). The linear MTF fringes during a snapshot are regularly spaced but the 
Table 1. Piston RMS and AO RMS specifications in fraction of the wavelength for a variation of $\approx 15$ per cent of the merit functions. $l_{\mathrm{c}}$ is the coherence length.

\begin{tabular}{lcccc}
\hline $\begin{array}{l}\text { Source of errors: } \\
\text { Exposure time: }\end{array}$ & $\begin{array}{c}\text { Piston } \\
\text { Short }\end{array}$ & $\begin{array}{c}\text { Piston } \\
\text { Long }\end{array}$ & $\begin{array}{c}\mathrm{AO} \\
\text { Short }\end{array}$ & $\begin{array}{c}\mathrm{AO} \\
\text { Long }\end{array}$ \\
\hline Interferometric Strehl & $l_{\mathrm{c}}$ & $\lambda / 8$ & $\lambda / 18$ & $\lambda / 18$ \\
Visibility & $l_{\mathrm{c}}$ & $\lambda / 12$ & $\lambda / 8$ & $\lambda / 6$ \\
$R_{23}$ quantity & $l_{\mathrm{c}}$ & $\lambda / 12$ & $\lambda / 18$ & $\lambda / 18$ \\
FWHM central peak & $l_{\mathrm{c}}$ & $l_{\mathrm{c}}$ & $\lambda / 18$ & $\lambda / 18$ \\
FWHM lateral peak & $l_{\mathrm{c}}$ & $l_{\mathrm{c}}$ & $\lambda / 10$ & $\lambda / 18$ \\
Intensity lateral peak & $l_{\mathrm{c}}$ & $\lambda / 10$ & $\lambda / 18$ & $\lambda / 24$ \\
\hline
\end{tabular}

fringe spacing reduces as the tip-tilt increases. Those fringes are perpendicular to the differential shift of the two envelopes in the PSF and to the differential tip-tilt direction in the entrance pupil. The differential tip-tilt amplitude and direction are therefore correlated - respectively - to the fringe spacing and orientation in the central MTF peak. The differential tip-tilt (e.g. due to pointing errors between both telescopes) can be instantaneously estimated by measuring the central peak widths. Indeed, the central peak FWHMs in the $\mathrm{X}$ and $\mathrm{Y}$ directions, respectively parallel and perpendicular to the baseline of the LBTI, behave almost the same but slightly differ when fringes occur in short MTF exposures (Fig. 7).

\subsection{Piston and $\mathrm{AO}$ correction requirements}

We have studied in details the effect of $\mathrm{AO}$ and piston errors on to the rings and fringes forming the PSF structure. The AO errors in short exposures affect first the contrast in the rings whereas the fringed speckles remain contrasted. The AO errors in long exposures attenuate rapidly the contrast in both the rings and fringes. The piston errors in short exposures do not damage the contrast in both fringes and rings. The piston errors in long exposures attenuate mainly the contrast in the fringes, while rings are rather unaffected. In other words, in short exposures, fringes can be partially recovered despite of $\mathrm{AO}$ errors and piston errors, but rings are rapidly destroyed by AO errors. In long exposures, fringes are affected by both $\mathrm{AO}$ and piston errors, whereas rings are disturbed only by $\mathrm{AO}$ errors.

The LBTI Fizeau imager is primarily limited by the AO performance and, as well, by the differential piston/tip-tilt errors occurring in both eyes of the LBTI. A loss of 15 per cent of the interferometric Strehl in the visible wavelengths at $750 \mathrm{~nm}$ occurs for AO errors higher than $\lambda / 18 \approx 40 \mathrm{~nm}$, or for piston errors higher than $\lambda / 8 \approx$ $100 \mathrm{~nm}$ (according to the interferometric Strehl) or $\lambda / 12 \approx 70 \mathrm{~nm}$ (accounting for the $R_{23}$ quantity). Both effects at a time reduce the interferometric Strehl to $0.85^{2}=72$ percent. Thus, to provide an image quality suitable for standard science cases (i.e. an interferometric Strehl above 70 percent), the LBTI Fizeau mode requires both at a time: an AO correction better than $\approx \lambda / 18$ RMS for both short and long exposures and a piston correction better than $\approx \lambda / 8$ RMS for long exposures or simply below the coherence length for short exposures.

To have comparable impact on the interferometric Strehl during a long exposure at a given wavelength, $\mathrm{AO}$ wavefront RMS errors need to be about twice as small as the differential piston RMS errors. This reflects the fact that the Fizeau mode combines two single apertures, so that the single aperture AO precision needs to be better than the piston correction. Actually, the $\mathrm{AO}$ requirement is tighter, but also easier to reach on-sky as it is only over a single 8-m aperture. On the contrary, more emphasis needs to be given to meet the piston specifications, requiring a sufficient dynamic control range for combining the telescopes at the long 23-m baseline. Therefore, in practice, the piston RMS requirement is lower but harder to meet than the AO RMS requirement.

\subsection{Piston, tip-tilt and AO correction at the LBTI}

The LBTI is equipped with efficient FLAO systems providing high Strehl performance (Esposito et al. 2010). In complement, a fast piston/tip-tilt mirror is used in the beam combiner of the LBTI to correct residual errors in real-time. The tip-tilt control requires a precision better than a fraction of the angular resolution of a sub-aperture $\lambda / D$, in order to stabilize the diffraction envelopes in the PSF produced by the two sub-images of each 8-m telescope. Individual tip-tilt is corrected by each AO system and amounts to 3-15 mas RMS at $\approx 50 \mathrm{~Hz}$, depending on the weather conditions and on the brightness of the star. However, the tip-tilt control done by each single telescope AO system is usually not enough. Indeed, the Fizeau mode is more sensitive to differential tip-tilt than to individual tip-tilt due to the long baseline. Thus, the differential tip-tilt is corrected by the fast tip-tilt mirror and reaches currently a few mas RMS at $1 \mathrm{kHz}$. Improving the tip-tilt accuracy requires some additional sensor which could be for instance an MTF fringe sensor (as proposed in Section 5.1).

The shift of the image due to tip-tilt is of the same order as the width of the Airy envelope in the visible $(\lambda / D=20$ mas in $I$ band) and is about 5 times smaller in the near-infrared $(\lambda / D=100$ mas in $L$ band). The piston control in closed-loop can attain $\approx 400 \mathrm{~nm}$ RMS (Defrère et al. 2014, 2016a,b), that corresponds to $\approx \lambda / 2$ in the visible $I$ band and $\approx \lambda / 10$ in the near-infrared $L$ band. The requirements are relaxed by a factor of $\approx 5$ in $L$ band $(\lambda=3.8$ $\mu \mathrm{m})$ compared to the $I$ band $(\lambda=0.76 \mu \mathrm{m})$. This make the LBTI Fizeau mode right now feasible in the near-infrared wavelengths, at least for the standard science case. However, for a visible mode, one of the main requirements would be to reduce both the piston and tip-tilt residuals. Alternatively, a burst mode with a lucky imaging approach shall deal with that, by discarding the frames having a significant differential tip-tilt.

\section{CONCLUSION}

In this paper, we have critically evaluated various merit functions used to characterize the PSFs and the MTFs of the LBTI Fizeau imager. The interferometric Strehl and the $R_{23}$ quantity appears as two relevant merit functions to describe the high-contrast imaging performance of a given aberrated wavefront, knowing the wavefront RMS provided by the two FLAO systems and the piston RMS provided by the LBTI combiner.

We have also estimate the high-level requirements against optodynamical aberrations for high-contrast imaging applications. The LBTI Fizeau imager can be used for high-contrast imaging, providing a high-Strehl regime (by both AO systems), a cophasing mode (by a fringe tracker) and a burst mode (by a fast camera) to record fringed speckles in short exposures. The Fizeau mode is primarily limited by the AO performance and, as well, by the differential piston/tip-tilt errors occurring in both eyes of the LBTI. The contrast can however be recovered against residual piston errors by acquiring short exposures at high frame rate (as long as the piston RMS remains below a fraction of the coherence length). During a long exposure, the performance is similarly affected if the AO residual RMS error is about twice as small as the differential piston 
RMS error. However, in practice, the piston RMS requirement is lower but harder to meet than the AO RMS requirement.

The LBTI facility is equipped with efficient FLAO systems and a fast piston/tip-tilt mirror. That is why the LBTI Fizeau mode can provide an image quality suitable for standard science cases (i.e. an interferometric Strehl above 70 percent) by performing both at a time: an $\mathrm{AO}$ correction better than $\approx \lambda / 18$ RMS $(\approx 40 \mathrm{~nm}$ in $I$ band, $\approx 200 \mathrm{~nm}$ in $L$ band) for both short and long exposures, and a piston correction better than $\approx \lambda / 8$ RMS $(\approx 100 \mathrm{~nm}$ in $I$ band, $\approx 500 \mathrm{~nm}$ in $L$ band) for long exposures or simply below the coherence length for short exposures. Such results, which can be applied to any observing wavelength, suggest that $\mathrm{AO}$ and piston control at the LBTI would already enable high-contrast imaging at near- and mid-infrared wavelengths.

The simulations described here can be further improved by developing a more sophisticated tool, but the results are relevant for a high-Strehl AO regime. We wish to pursue this study in order to fairly compare the performance of the LBTI Fizeau mode to the one of dedicated high-contrast missions (e.g. FLAO, SPHERE, GPI). For this aim, in a forthcoming paper, we will develop an end-to-end simulation tool by modelling science cases (e.g. binary, exoplanet, etc.) and realistic aberrations (e.g. residual piston/tiptilt fluctuations due to the atmosphere and to the vibrations, widefield AO errors, pointing errors, misalignments, spectral bandwidth, etc.) and by using post-processing techniques (e.g. frames selection, lucky imaging, shift-and-add, deconvolution, ADI mode, etc.).

\section{REFERENCES}

Angel J. R. P., Woolf N., 1997, in Soderblom D., ed, ASP Conf. Ser. Vol. 119, Planets Beyond the Solar System and the Next Generation of Space Missions. Astron. Soc. Pac., San Francisco, p. 207

Angel J. R. P., Hill J. M., Strittmatter P. A., Salinari P., Weigelt G., 1998, in Reasenberg R. D., ed., Proc. SPIEConf. Ser. Vol. 3350, Astronomical Interferometry. SPIE, Bellingham, p. 881

Bailey V. P. et al., 2014, in Marchetti E., Close L. M., Véran J.-P., eds, Proc. SPIE Conf. Ser. Vol. 9148, Adaptive Optics Systems IV. SPIE, Bellingham, p. 914803

Böhm M., Pott J.-U., Sawodny O., Herbst T., Kürster M., 2014, MNRAS, 442, 2446

Böhm M. et al., 2016, in Hall H. J., Gilmozzi R., Marshall H. K, eds, Proc. SPIE Conf. Ser. Vol. 9906, Ground-based and Airborne Telescopes VI. SPIE, Bellingham, p. 99062R

Buscher D. F. et al., 1995, Appl. Opt., 34, 1081

Defrère D. et al., 2014, in Rajagopal J. K., Creech-Eakman M. J., Malbet F., eds, Proc. SPIE Conf. Ser. Vol. 9146, Optical and Infrared Interferometry IV. SPIE, Bellingham, p. 914609

Defrère D. et al., 2016a, ApJ, 824, 66
Defrère D. et al., 2016b, Proc. SPIE, 9907, 99071G

Egner S. E., Herbst T. M., Arcidiacono C., 2008, in Schöller M., Danchi W. C., Delplancke F., eds, Proc. SPIE Conf. Ser. Vol. 7013, Optical and Infrared Interferometry. SPIE, Bellingham, p. 70133C

Esposito S. et al., 2010, Proc. SPIE, 7736, 773609

Glück M., Pott J.-U., Sawodny O., 2017, PASP, 129, 065001

Herbst T., 2003, Ap\&SS, 286, 45

Herbst T. M., Ragazzoni R., Eckart A., Weigelt G., 2004, in Moorwood A. F. M., Iye M., eds, Proc. SPIE Conf. Ser. Vol. 5492, Ground-based Instrumentation for Astronomy. SPIE, Bellingham, p. 1045

Herbst T. M., Ragazzoni R., Eckart A., Kurster M., 2005, LINC-NIRVANA Error Budget, Doc. No. LN-MPIA-FDR-GEN-001

Herbst T. M., Ragazzoni R., Eckart A., Weigelt G., 2008, in Schöller M., Danchi W. C., Delplancke F., eds, Proc. SPIE Conf. Ser. Vol. 7013, Optical and Infrared Interferometry. SPIE, Bellingham, p. 701326

Hill J. M., Ashby D. S., Brynnel J. G., Christou J. C., Little J. K., Summers D. M., Veillet C., Wagner R. M., 2014, in Larry M., Stepp L. M., Gilmozzi R., Hall H. J., eds, Proc. SPIE Conf. Ser. Vol. 9145, Ground-based and Airborne Telescopes V. SPIE, Bellingham, p. 914502

Hinz P. et al., 2012, in Delplancke F., Rajagopal J. K., Malbet F., eds, Proc. SPIE Conf. Ser. Vol. 8445, Optical and Infrared Interferometry III. SPIE, Bellingham, p. $84450 \mathrm{U}$

Hinz P. et al., 2014, in Rajagopal J. K., Creech-Eakman M. J., Malbet F., eds, Proc. SPIE Conf. Ser. Vol. 9146, Optical and Infrared Interferometry IV. SPIE, Bellingham, p. 914605

Hinz P. M. et al., 2016. in Malbet F., Creech-Eakman M. J., Tuthill P. G., eds, Proc. SPIE Conf. Ser. Vol. 9907, Optical and Infrared Interferometry and Imaging V. SPIE, Bellingham, p. 990704

Labeyrie A., 1970, A\&A, 6, 85

Leisenring J. M. et al., 2012, in McLean I. S., Ramsay S. K., Takami H., eds, Proc. SPIE Conf. Ser. Vol. 8446, Ground-based and Airborne Instrumentation for Astronomy IV. SPIE, Bellingham, p. 84464F

McCarthy D. W., Sabatke E. M., Sarlot R. J., Hinz P. M., Burge J. H., 2000, in Léna P., Quirrenbach A., eds, Proc. SPIE Conf. Ser. Vol. 4006, Interferometry in Optical Astronomy. SPIE, Bellingham, p. 659

Noll R. J., 1976, J. Opt. Soc. Am., 66, 207

Patru F., Esposito S., Puglisi A., Riccardi A., Pinna E., Arcidiacono C., Hill J., Hinz P., 2016a, in Malbet F., Creech-Eakman M. J., Tuthill P. G., eds, Proc. SPIE Conf. Ser. Vol. 9907, Optical and Infrared Interferometry and Imaging V. SPIE, Bellingham, p. 99071S

Patru F., Esposito S., Puglisi A., Riccardi A., Pinna E., Arcidiacono C., Hill J., Hinz P., 2016b, in Malbet F., Creech-Eakman M. J., Tuthill P. G., eds, Proc. SPIE Conf. Ser. Vol. 9907, Optical and Infrared Interferometry and Imaging V. SPIE, Bellingham, p. 99071T

Patru F. et al., 2017, MNRAS, in press

This paper has been typeset from a $\mathrm{T}_{\mathrm{E}} \mathrm{X} / \mathrm{L} \mathrm{T} \mathrm{EX}$ file prepared by the author. 